\title{
MaxEnt's parameter configuration and small samples: Are we paying attention to recommendations? A systematic review
}

\author{
Narkis S Morales ${ }^{\text {Corresp., }}{ }^{1,2}$ ， Ignacio C Fernández ${ }^{2,3}$, Victoria Baca-González ${ }^{4}$ \\ 1 Department of Biological Sciences, Faculty of Science and Engineering, Macquarie University, Sydney, New South Wales, Australia \\ 2 Fundación Ecomabi, Santiago, Región Metropolitana, Chile \\ 3 Landscape Ecology \& Sustainability Laboratory, Arizona State University, Arizona State University, Tempe, Arizona, United States \\ 4 Facultad de Ciencias Biológicas, Universidad Complutense de Madrid, Madrid, Spain \\ Corresponding Author: Narkis S Morales \\ Email address: narkis.moralessanmartin@mq.edu.au
}

Environmental niche modeling (ENM) is commonly used to develop probabilistic maps of species distribution. Among available ENM techniques, MaxEnt has become one of the most popular tools for modeling species distribution, with hundreds of peer-reviewed articles published each year. MaxEnt's popularity is mainly due to the use of a graphical interface and automatic parameter configuration capabilities. However, recent studies have shown that using the default automatic configuration may not be always appropriate because it can produce non-optimal models; particularly when dealing with a small number of species presence points. Thus, the recommendation is to evaluate the best potential combination of parameters (feature classes and regularization multiplier) to select the most appropriate model. In this work we reviewed 244 articles from 142 journals between 2013 and 2015 to assess whether researchers are following recommendations to avoid using the default parameter configuration when dealing with small sample sizes, or if they are using MaxEnt as a "black box tool". Our results show that in only $16 \%$ of analyzed articles authors evaluated best feature classes, in $6.9 \%$ evaluated best regularization multipliers, and in a meager $3.7 \%$ evaluated simultaneously both parameters before producing the definitive distribution model. We analyzed 20 articles to quantify the potential differences in resulting outputs when using software default parameters instead of the alternative best model. Results from our analysis reveal important differences between the use of default parameters and the best model approach, especially in the total area identified as suitable for the assessed species and the specific areas that are identified as suitable by both modelling approaches. These results are worrying, because publications are potentially reporting over-complex or over-simplistic models that can undermine the applicability of their results. Of particular importance are studies used to inform policy making. Therefore, researchers, practitioners, reviewers and editors need to be very judicious when dealing with MaxEnt, particularly when the modelling process is 
based on small sample sizes. 


\section{MaxEnt's parameter configuration and small samples: Are we paying attention to recommendations? A systematic review}

3

4 Narkis S. Morales ${ }^{\mathrm{a}, \mathrm{c}}$, Ignacio C. Fernández ${ }^{\mathrm{b}, \mathrm{c},{ }^{*} \text { and Victoria Baca-González }}$.

5

$6 \quad{ }^{a}$ Department of Biological Sciences, Faculty of Science and Engineering, Macquarie University, Building E8B

7 Room 206, NSW 2109, Sydney, Australia. (email: narkis.moralessanmartin@mq.edu.au)

8 bLandscape Ecology \& Sustainability Laboratory, Arizona State University, LSE Room 704, Tempe, AZ 85281,

$9 \quad$ USA. (email: ignacio.fernandez@asu.edu).

10 'Fundación Ecomabi, Ahumada 312, Oficina 425, 8320185 Santiago, Chile.

11 dFacultad de Ciencias Biológicas, Universidad Complutense de Madrid, José Antonio Novais 12, Ciudad

12 Universitaria, Madrid 28040, Spain. (email: vbaca01@ucm.es )

13

$14 *$ Corresponding author

15

16 


\section{Abstract}

18 Environmental niche modeling (ENM) is commonly used to develop probabilistic maps of species distribution. Among available ENM techniques, MaxEnt has become one of the most popular tools for modeling species distribution, with hundreds of peer-reviewed articles published each year. MaxEnt's popularity is mainly due to the use of a graphical interface and automatic parameter configuration capabilities. However, recent studies have shown that using the default automatic configuration may not be always appropriate because it can produce nonoptimal models; particularly when dealing with a small number of species presence points. Thus, the recommendation is to evaluate the best potential combination of parameters (feature classes and regularization multiplier) to select the most appropriate model. In this work we reviewed 244 articles from 142 journals between 2013 and 2015 to assess whether researchers are following recommendations to avoid using the default parameter configuration when dealing with small sample sizes, or if they are using MaxEnt as a "black box tool". Our results show that in only $16 \%$ of analyzed articles authors evaluated best feature classes, in $6.9 \%$ evaluated best regularization multipliers, and in a meager $3.7 \%$ evaluated simultaneously both parameters before producing the definitive distribution model. We analyzed 20 articles to quantify the potential differences in resulting outputs when using software default parameters instead of the alternative best model. Results from our analysis reveal important differences between the use of default parameters and the best model approach, especially in the total area identified as suitable for the assessed species and the specific areas that are identified as suitable by both modelling approaches. These results are worrying, because publications are potentially reporting overcomplex or over-simplistic models that can undermine the applicability of their results. Of particular importance are studies used to inform policy making. Therefore, researchers, 
40 practitioners, reviewers and editors need to be very judicious when dealing with MaxEnt,

41 particularly when the modelling process is based on small sample sizes.

42

\section{Introduction}

Environmental niche modeling (ENM), also referred as to predictive habitat distribution modeling (e.g. Guisan \& Zimmermann, 2000), or species distribution modeling (e.g. Elith \& Leathwick, 2009; Miller, 2010), is a common technique increasingly used in a variety of disciplines interested in the geographical distribution of species. ENMs have been used, among other disciplines, in landscape ecology (Amici et al., 2015), biogeography (Carvalho \& Del Lama, 2015), conservation biology (Bernardes et al., 2013, Brambilla et al., 2013), marine sciences (Bouchet \& Meeuwig, 2015; Crafton, 2015), paleontology (Stigall \& Brame, 2014), plant ecology (Gelviz-Gelvez et al., 2015), public health (Ceccarelli \& Rabinovich, 2015) and restoration ecology (Fernandez \& Morales, 2016).

The basic principle behind the ENM is the use of environmental information layers and species presence, pseudo-absence or absence points to develop probabilistic maps of distribution suitability (Elith \& Leathwick, 2009). ENMs are generally used for four main objectives: (1) to estimate the relative suitability of the habitat currently occupied by assessed species, (2) to estimate the relative suitability of habitat in areas where assessed species are currently not known to be present, (3) to estimate potential changes in the suitability of habitat due to environmental change scenarios, and (4) to estimate the species environmental niche (Warren \& Seifert, 2011). 
63 Among the available tools for ENM, the maximum entropy approach is one of the most widely

64 used for predicting species distributions (Fitzpatrick et al., 2013; Merow et al., 2013). The

65 maximum entropy approach, part of the family of the machine learning methods, is currently

66 available in the software MaxEnt (Phillips et al., 2006;

67 https://www.cs.princeton.edu/ schapire/maxent/). MaxEnt can model potential species

68 distributions by using a list of species presence-only locations and a set of environmental

69 variables (Elith et al., 2010). Since 2004 the use of MaxEnt has grown exponentially (Figure 1).

70 Nowadays MaxEnt is one of the preferred methods used for predicting potential species

71 distribution among researchers (Merow et al., 2013).

72

73 The simplicity and straightforward steps required to run MaxEnt seem to have tempted many

74 researchers to use it as a black box despite the increasing evidence that using MaxEnt with

75 default parameter settings (i.e. auto-features) will not necessarily generate the best model (e.g.

76 Shcheglovitova \& Anderson, 2013; Syfert et al., 2013; Radosavljevic \& Anderson, 2014).

77 MaxEnt has two main modifiable parameters: (1) feature classes and (2) regularization

78 multiplier. Feature class corresponds to a mathematical transformation of the different covariates

79 used in the model to allow complex relationship to be modeled (Elith et al., 2010). The

80 regularization multiplier is a parameter that adds new constraints, in other words is a penalty

81 imposed to the model. The main goal is to prevent over-complexity and/or overfitting by

82 controlling the intensity of the chosen feature classes used to build the model (Elith et al., 2010;

83 Shcheglovitova \& Anderson, 2013). We recommend look at Merrow et al. (2013) for a detailed

84 explanation of features and regularization multipliers. 
86 Some authors have argued that the use of default parameters without providing information on

87 this decision could mean that several of published results could be based on over-complex or

over-simplistic models (Warren \& Seifert, 2011; Cao et al., 2013; Merrow et al., 2013). For example, Anderson \& Gonzalez (2011) compared different MaxEnt configurations to determine the optimal configuration that minimizes overfitting. Their results showed that in several cases the optimal regularization multiplier was not the default. This is supported by other studies showing that a particular combination of feature classes and regularization multiplier provided better results than the default settings (Syfert et al., 2013), and that the default configuration provided by MaxEnt is not necessarily the most appropriate, especially when dealing with small samples size (Warren \& Seifert, 2011; Shcheglovitova \& Anderson, 2013).

Whereas several authors have highlighted the potential problems of models generated by MaxEnt default settings and provided recommendation to deal with this issue (e.g. Warren \& Seifert, 2011; Merow et al., 2013; Yackulic et al, 2013; Halvorsen et al., 2015), there is no information regarding the echo that these recommendations have had on current MaxEnt use, and neither on how this could be affecting published results. Aiming to answer these questions, in this work we aimed: First, to evaluate if researchers are paying attention to recommendations regarding the importance of evaluating the best potential combination of MaxEnt's parameters for modelling species distribution. Second, to quantify the potential differences in resulting outputs when using MaxEnt default parameters instead of evaluating different sets of parameters combinations to identify an alternative best model. To achieve our first objective we review and analyze the published literature from years 2013 to 2015, focusing our analysis in the modelling information provided by articles reporting results based on small numbers of species presence points (i.e. less 
109 than 90 presence points). For the second objective, we selected from our review results a sample

110 of 20 case studies, and we performed the modelling process using default setting and a

111 combination of parameters to assess the differences between the default and the alternative best

112 model outputs.

113

114 Materials and Methods

115

116 Literature analysis

117

118 We used our own literature search protocol using the databases available through the ISI Web of

119 Science (ISI WOS; http://webofknowledge.com/) search engine (S1) by using the keywords

120 "MaxEnt" and "species distribution" in the topic. Because many of the recommendations were

121 published between 2011 and 2012, we restricted our search to the 2013-2015 period, assuming

122 that if researchers were alert to recommendations these changes would be noticed on

123 publications of following years. The search was carried out by by N.S Morales and V. Baca-

124 González during the months of March and April, 2016. Whereas we only used English key

125 words for our search, we also included in our analysis the articles published in Spanish and

126 Portuguese but with abstracts written in English. From these results we only selected studies

127 reporting $\leq 90$ presence species points for the modelling process. We chose this threshold value

128 because major changes in MaxEnt auto-features parameters occurs when less than 80 presence

129 records points are used for modelling (Phillips \& Dudík, 2008; Merrow et al., 2013), implying

130 that a sample of 90 could easily represent less than 80 presence points for modelling due to the

131 required sample points that needs to be set aside for validation purposes. Because for some 
132 authors the $\leq 90$ presence species points threshold may be considered rather large for defining

133 what a small sample size is (e.g. Phillips \& Dudík, 2008; Shcheglovitova \& Anderson, 2013), we

134 attempted to overcome this potential issue by ensuring that half of the case studies (i.e. 10) used

135 for performing our modeling analysis had less than 15 presence points.

136

137 Our preliminary literature search yielded 816 articles. From these articles, 244 reported a sample 138 size of $\leq 90$ presence points and were therefore used for our literature analyses (Figure 2, Table 139 1, see the detailed articles list in S2). Any doubt or disagreement in the classification of the 140 articles was discussed with I.C. Fernández; whose opinion was taken as final decision. We 141 reviewed the methodological information provided in the selected articles to determine the types 142 of feature classes and regularization multiplier used for modelling process. We classified features 143 and regularization multiplier used in each paper in three main categories: (1) user-defined 144 parameters, (2) software default parameters, (3) and no information provided. We also evaluated 145 if the articles provided data on the geographical coordinates of presence points used for the 146 modelling process (i.e. lists of georeferenced presence points or species presence maps), which

147 we considered a fundamental input for performing the modelling process. We considered only 148 those articles providing information on features, regularization multiplier and geographical 149 coordinates as suitable for modelling analysis.

\section{Modelling Analysis}

152

153 To quantify the potential differences in resulting outputs when using software default parameters

154 instead of different parameters combinations to identify an alternative best model, we first 
155 generated a list consisting on all publications providing the geographical locations of presence

156 points used for modelling and that report having used default parameters (feature classes and

157 regularization multiplier). These selected publications were sorted in two groups, those with less

158 than 15, and those between 16 and 90 sample presence points. From each of these groups we

159 randomly selected 10 articles for our analysis. If the selected articles in any of the two groups

160 were considered too similar in terms of the number of samples and area of analysis (extent), we

161 repeated the process until having a heterogeneous sample that increases the strength of our

162 analysis. With this we aimed to include studies from different regions, with varying geographical

163 extents, and differing number of species presence points. For each of these articles we collected

164 the geographical coordinates of species presence points and performed the modelling process

165 using default features, and a set of 72 different parameter combinations, aiming to quantify

166 potential differences on resulting outputs when using default parameters instead of analyzing an

167 alternative best model. For all our modelling we used the WorldClim database

168 (http://www.worldclim.org) as our environmental variables dataset, standardizing all the analysis

169 to a $\sim 1 \mathrm{~km}^{2}$ resolution grid. To select the best model parameters we compared different models

170 with a combination of the "feature class" and "regularization multiplier". MaxEnt provides

171 different types of restrictions ("feature class") in the modelling stage such as lineal (L), quadratic

172 (Q), product $(\mathrm{P})$, threshold $(\mathrm{T})$, and hinge $(\mathrm{H})$. We used all the possible combinations of these

173 features (12 combinations). The used regularization multiplier values were based on Warren and

174 Seifert (2011) and Shcheglovitova \& Anderson (2013): 1, 2, 5, 10, 15, and 20. Combining

175 features classes and regularization multipliers, we assessed a total of 72 models for each case

176 study, plus the default auto-feature. For each case of study we selected the "best model" by using

177 the $\mathrm{AIC}_{\mathrm{c}}$ criterion, as this model selection criterion outperforms other available criterion (e.g. 
178 AUC) for comparing different models generated through MaxEnt, particularly for small sample 179 sizes (Warren and Seifert, 2011). A detailed description of the methods used for modelling is 180 provided in $\mathrm{S} 3$.

\section{Results}

\section{Literature analysis}

From the 244 articles that reported a sample size $\leq 90$ for the $2013-2015$ period, $44.0 \%$ (108 articles) did not provide information about the features used for modelling, $40.0 \%$ (97 articles) reported to have used default features, and only 16.0\% (39 articles) reported to have used userdefined features (Figure 3; S2). In terms of the regularization multiplier, 48.8\% (119 articles) did not provide any information about the regularization multiplier used for modelling, 43.4\% (106 articles) used the default regularization multiplier, and only 6.9\% (19 articles) reported having used a user-defined regularization multiplier (Figure 3; S2). Considering both default parameters, merely 3.7\% (9 articles) of the reviewed articles reported having used user-defined settings for both parameters (S2).

Even though $70.5 \%$ (172 articles) of publications provide a list or a map with the geographical coordinates of the presence points used for modelling, and 47.1\% (115 articles) reported both feature classes and regularization multipliers used for modelling; only $34.3 \%$ ( 84 articles) of the analyzed publications provide all three elements together (Figure 4). 
201

202

203

204

205

206

207

208

209

210

211

212

213

214

215

216

217

218

219

220

221

222

223

\section{Modelling analysis}

Results from our modelling analysis reveal huge potential effects of using a default parameter instead of a best model approach for identifying best suitable areas for species distribution (Table

2). Although our results show that the spatial correlation between default and best model outputs is relatively high, and that fuzzy kappa statistics show high similarity between generated maps for all assessed case studies, the total area identified as suitable for the assessed species tend to greatly differ, particularly for species covering large geographical extents (Table 2). Nevertheless, we did not find statistical signs that suggest that outputs generated by defaults setting tend to predict larger or smaller total suitable areas than the alternative best model $(\mathrm{p}=$ 0.093, paired t-test for log transformed variables). However, it is not only the difference on total suitable area that differs, but also the specific areas that are identified as suitable by both modelling approaches (i.e. shared area). Whereas in average the proportion of shared areas tend to be considerably larger than the not-shared area (mean shared area ratio $=2.483$ ), our data shows that for some cases there could be large discrepancies, with the majority of predicted suitable areas not overlaying between model outputs (i.e. shared ratio $<1$ ). (Table 2).

列

The sample size (i.e. number of presence points) seems to not affect the degree of differences between the outputs obtained by using the default setting or by evaluating a set of parameters to select an alternative best model. In fact, our analysis show that sample size does not affect the spatial correlation $\left(\mathrm{R}^{2}=0.026, \mathrm{p}=0.501\right)$, fuzzy kappa $\left(\mathrm{R}^{2}=0.005, \mathrm{p}=0.770\right)$, or shared $/$ not shared ratio $\left(\mathrm{R}^{2}=0.004, \mathrm{p}=0.786\right)$ between modelling outputs. Also our results do not show any trend showing that sample size may favor the selection of some parameter combination over 
224 others, bedside the fact that for all study cases the best models tend to be associated to small

225 regularization multipliers (Table 2). These results highlight the importance of evaluating what

226 combination of parameters could provide the best modelling results, independently of the sample

227 size used for modelling.

228

229 Discussion

230

231

Are we paying attention to recommendations?

232

233

Whereas there is increasing evidence that the use of MaxEnt default parameters do not always generate the best possible model output (e.g. Syfert et al., 2013; Radosavljevic \& Anderson, 2014), and different authors have highlighted the importance to evaluate the best combination of these parameters before deciding on the best model (see Anderson \& Gonzalez, 2011; Warren \& Seifert, 2011), results from our analysis indicate that researchers have been rather indifferent to these recommendations. In fact, our literature analysis shows that the use of MaxEnt default parameters for modelling species distribution with small recorded presence points seems to be the rule rather than the exception. More than $40 \%$ of the articles analyzed in our study do not provide information about the parameters configuration used to run the models, which reveals the little attention that researchers and reviewers are paying to this specific issue. Our results also reveal that among the articles that do provide information about the features and regularization multiplier used, a large proportion reported to have used the software default configuration. This preference towards using default setting has remained strong despite the variety of articles describing how MaxEnt works and should be used (Phillips \& Dudík, 2008), the proper 
248 best parameters combination (e.g. Anderson \& Gonzalez, 2011; Warren \& Seifert, 2011; Syfert

249 et al., 2013; Radosavljevic \& Anderson, 2014) and the increasing publication of approaches to

250 select the best model by using appropriate parameters combinations (see Anderson \& Gonzalez,

251 2011; Syfert et al., 2013; Shcheglovitova \& Anderson, 2013).

252

253 We did not observe any trend in the data that would suggest a change from "black box" users

254 towards the use of user-defined parameters. Although our reviewed articles cover a relatively

255 short period of time (2013-2015), if authors were inclined to adopt best practices for modelling

256 we would have expected to see a trend in the data showing an increasing use of user-defined

257 features over time. However, the only trend in our results is the increasing number of articles not

258 providing information on the features and regularization multiplier used for modelling. We do

259 not have a clear explanation for this trend, but we believe that it is probably due to new

260 researchers using the modelling software without paying proper attention to current MaxEnt

261 literature, particularly to the publications referring to the importance of analyzing parameters

262 combination for selecting the best model (e.g. Anderson \& Gonzalez, 2011; Warren \& Seifert,

263 2011; Syfert et al., 2013; Radosavljevic \& Anderson, 2014).

264

265 The widespread use of default parameters is not the only caveat we found in our literature 266 analysis. We also found a general lack of information that would allow for replicating, assessing 267 or comparing the results from published studies. This information is not only relevant in terms of 268 potential replication of the research, but also necessary for reviewers to evaluate if the outputs 269 from the modelling process are reliable, or are affected among other factors by parameters used, 270 unreliable species presence data sources, or geographically biased presence points records. 
272 Whereas in our literature review we limited the search of articles only to the ISI WOS database,

273 this database includes the large majority of mainstream journals dealing with species distribution

274 modelling (S2) and is often regarded as including journals with high quality standards. Therefore

275 we consider that our results are a robust representation of the current lack of attention to recent

276 published recommendations on how to better use MaxEnt.

277

\section{Implications for research and practice}

279

280

There are no doubts of the huge potential that MaxEnt has for helping understanding species

281

282

283

284

285

286

287

288

289

290

291

292

293

distribution and for its application as a decision-making tool, which is reflected by the large diversity of disciplines that currently are using it. Nevertheless, as any modelling approach, results obtained through MaxEnt will largely depend on the quality of input data (i.e. reliability of environmental and species presence data) (Yackulic et al. 2013) and parameterization used for modelling (Warren \& Seifert, 2011; Cao et al., 2013; Merrow et al., 2013). Whereas in this work we did not evaluate if the input data used for modelling could be considered reliable or appropriate, it is important to take into account that results can be largely affected by species presence sampling bias (Kramer-Schadt et al., 2006; Syfert et al., 2013; Yackulic et al. 2013) and by the geographical extent used for modelling (Merow et al., 2013).

For the case of parameterization (i.e. combination of features and regularization multiplier), results from our case studies strongly support the claims made by previous studies in relation that using MaxEnt default parameters may not generate the best results (e.g. Anderson \& Gonzalez, 
294 2011; Warren \& Seifert, 2011; Syfert et al., 2013; Radosavljevic \& Anderson, 2014). In fact in

295 none of the 20 case studies analyzed in our work the model generated by using default

296 parameters were selected as the best model, which is a worrying sign because an important

297 proportion of MaxEnt published literature can be presenting modelling outputs based on over-

298 simplistic or over-complex models. In other words, reported models can be overestimating the

299 potential distribution of assessed species, or overfitting modelling output to the input data, 300 therefore losing its ability to identify the optimal range of environmental conditions that are 301 suitable for the species (Warren \& Seifert 2011; Merow et al, 2013).

302

303 Nevertheless, perhaps the most relevant implications of an inadequate use of MaxEnt for 304 modelling species distribution are on the decision-making arena. When results from the 305 modelling processes are used directly to assess species conservation or to develop conservation 306 strategies, the areas identified as suitable for a given species could differ greatly depending on 307 the parameters used for modelling (Anderson \& Gonzalez, 2011). Whereas for our study cases 308 we only used environmental variables gathered from the WordClim database, and therefore our 309 models do not necessary replicate the results published by all the assessed studies, our results do 310 show that independently of the sample size, geographical region and extent of analysis, decisions 311 taken based on models generated by MaxEnt default setting could be strikingly different from 312 those taken based on the best model.

314 Conclusions and recommendations 
316 Results from our study may have vast implications, particularly with regard how articles are

317 being reviewed, and the replicability and transferability of the results. We adhere to the calls

318 from other authors to pay better attention to the potential implication of using Maxent's default

319 parameters when modelling species distribution, but we also suggest reviewers to carefully

320 evaluate if the methodological approach used for modelling is reliable and well supported in

321 recent literature. In addition, researchers need to provide as much information as possible to

322 allow proper evaluation and increase the potential replicability and transferability of their results.

Despite the fact that there are several studies that already include several recommendations how

to use and set up MaxEnt (e.g. Elith et al., 2006; Warren \& Seifert, 2011; Merow et al., 2013;

Yackulic et al, 2013; Halvorsen et al., 2015) we will try to summarize the most important points

that researchers need to keep in mind for selecting the best model from the set of potential outputs generated by changing features and regularization parameters.

During the process of building the model, the authors need to determine the best possible model using an objective methodology. One approach is the use of a jackknife procedure similar to the one describe by Shcheglovitova and Anderson (2013). The process consist in comparing different models with a combination of the parameters, "feature class" and "regularization multiplier" (see Shcheglovitova and Anderson (2013), Warren and Seifert (2011) and S3 for examples). The comparison of models can be done using the corrected Akaike information criterion (AICc) available in the software ENMTOOLS version 1.4.4 (Warren et al. 2011). The best model will correspond to the combination of "feature class" and "regularization multiplier" with the smallest AICc value. Although this is the methodology that we used in this work there 
339 are other methods that can be used. Another option is using a correlation analysis of the model-

340 predicted probabilities of occurrence and presences and absences proposed by Syfert et al.

341 (2013) or comparing the different map outputs using the fuzzy kappa statics based on Mestre et

342 al. (2015).

343

344 Once the best model is selected, replication of the best model (several runs; $n=30$ ) is needed to 345 determine that the results are consistent. Also, it is highly recommendable to validate the model 346 output using in situ surveys especially in cases that small numbers of occurrences were used to 347 generate the model. Although, we understand that this could be a major task when modelling 348 large extensions of habitat or rare species distributions; these limitations must be included in the 349 discussion and used with caution especially for management purposes.

350

351 These simple recommendations can help to improve the applicability of resulting models, which 352 in turn will help practitioners and decision-makers to use them more effectively as practical tools 353 for the development of management and conservation activities. While the use of MaxEnt's 354 default parameter can be very useful for having a quick picture of the potential distribution of a 355 given species, taking the necessary time to evaluate which parameters combination results in the 356 best model could largely increase the accuracy and reliability of modelling results. 


\section{References}

Amici, V., Eggers, B., Geri, F. \& Battisti, C. (2015) Habitat Suitability and Landscape Structure: A Maximum Entropy Approach in a Mediterranean Area. Landscape Research, 40, 208225.

Anderson, R.P. \& Gonzalez Jr, I. (2011) Species-specific tuning increases robustness to sampling bias in models of species distributions: An implementation with Maxent. Ecological Modelling, 222, 2796-2811.

Bernardes, M., Rödder, D., Nguyen, T.T., Pham, C.T., Nguyen, T.Q. \& Ziegler, T. (2013) Habitat characterization and potential distribution of Tylototriton vietnamensis in northern Vietnam. Journal of Natural History, 47, 1161-1175.

Bouchet, P.J. \& Meeuwig, J.J. (2015) Drifting baited stereo-videography: a novel sampling tool for surveying pelagic wildlife in offshore marine reserves. Ecosphere, 6, 1-29.

Brambilla, M., Bassi, E., Bergero, V., Casale, F., Chemollo, M., Falco, R., Longoni, V., Saporetti, F., Viganò, E. \& Vitulano, S. (2013) Modelling distribution and potential overlap between Boreal Owl Aegolius funereus and Black Woodpecker Dryocopus martius: implications for management and monitoring plans. Bird Conservation International, 23, 502-511.

Cao, Y., DeWalt, R.E., Robinson, J.L., Tweddale, T., Hinz, L. \& Pessino, M. (2013) Using Maxent to model the historic distributions of stonefly species in Illinois streams: The effects of regularization and threshold selections. Ecological Modelling, 259, 30-39.

Carvalho, A.F. \& Del Lama, M.A.D. (2015) Predicting priority areas for conservation from historical climate modelling: stingless bees from Atlantic Forest hotspot as a case study. Journal of Insect Conservation, 19, 581-587.

Ceccarelli, S. \& Rabinovich, J.E. (2015) Global Climate Change Effects on Venezuela's Vulnerability to Chagas Disease is Linked to the Geographic Distribution of Five Triatomine Species. Journal of Medical Entomology, 52, 1333-1343.

Crafton, R.E. (2015) Modeling invasion risk for coastal marine species utilizing environmental and transport vector data. Hydrobiologia, 746, 349-362.

Elith, J., H. Graham, C., P. Anderson, R., Dudík, M., Ferrier, S., Guisan, A., J. Hijmans, R., Huettmann, F., R. Leathwick, J., Lehmann, A., Li, J., G. Lohmann, L., A. Loiselle, B., Manion, G., Moritz, C., Nakamura, M., Nakazawa, Y., McC. M. Overton, J., Townsend Peterson, A., J. Phillips, S., Richardson, K., Scachetti-Pereira, R., E. Schapire, R., Soberón, J., Williams, S., S. Wisz, M., E. Zimmermann, N.(2006) Novel methods improve prediction of species' distributions from occurrence data. Ecography 29, 129151.

Elith, J. \& Leathwick, J.R. (2009) Species Distribution Models: Ecological Explanation and Prediction Across Space and Time. Annual Review of Ecology, Evolution, and Systematics, 40, 677-697.

Elith, J., Kearney, M. \& Phillips, S. (2010) The art of modelling range-shifting species. Method Ecol Evol, 1, 330 - 342.

Fernández, I.C. \& Morales, N.S. (2016) A spatial multicriteria decision analysis for selecting priority sites for plant species restoration: a case study from the Chilean biodiversity hotspot. Restoration Ecology, 24, 599-608.

Fitzpatrick, M.C., Gotelli, N.J. \& Ellison, A.M. (2013) MaxEnt versus MaxLike: empirical comparisons with ant species distributions. Ecosphere, 4, 1-15. 
402

403

404

405

406

407

408

409

410

411

412

413

414

415

416

417

418

419

420

421

422

423

424

425

426

427

428

429

430

431

432

433

434

435

436

437

438

439

440

Gelviz-Gelvez, S.M., Pavón, N.P., Illoldi-Rangel, P. \& Ballesteros-Barrera, C. (2015) Ecological niche modeling under climate change to select shrubs for ecological restoration in Central Mexico. Ecological Engineering, 74, 302-309.

Guisan, A. \& Zimmermann, N. (2000) Predictive habitat distribution models in ecology. Ecol Model, 135, 147 - 186.

Kramer-Schadt, S., Niedballa, J., Pilgrim, J. D., Schröder, B., Lindenborn, J., Reinfelder, V., ... \& Cheyne, S. M. (2013). The importance of correcting for sampling bias in MaxEnt species distribution models. Diversity and Distributions, 19, 1366-1379.

Merow, C., Smith, M.J. \& Silander, J.A. (2013) A practical guide to MaxEnt for modeling species' distributions: what it does, and why inputs and settings matter. Ecography, 36, 1058-1069.

Miller, J. (2010) Species Distribution Modeling. Geography Compass, 4, 490-509.

Moher, D., Liberati, A., Tetzlaff, J., Altman, D.G., The PRISMA Group (2009). Preferred Reporting Items for Systematic Reviews and Meta-Analyses: The PRISMA Statement. PLoS Med 6, e1000097.

Phillips, S.J. \& Dudík, M. (2008) Modeling of species distributions with Maxent: new extensions and a comprehensive evaluation. Ecography, 31, 161-175.

Phillips, S.J., Anderson, R.P. \& Schapire, R.E. (2006) Maximum entropy modeling of species geographic distributions. Ecological Modelling, 190, 231-259.

Radosavljevic, A. \& Anderson, R.P. (2014) Making better Maxent models of species distributions: complexity, overfitting and evaluation. Journal of Biogeography, 41, 629643.

Shcheglovitova, M. \& Anderson, R.P. (2013) Estimating optimal complexity for ecological niche models: A jackknife approach for species with small sample sizes. Ecological Modelling, 269, 9-17.

Stigall, A.L. \& Brame, H.-M.R. (2014) Relating environmental change and species stability in Late Ordovician seas. GFF, 136, 249-253.

Syfert, M.M., Smith, M.J. \& Coomes, D.A. (2013) The Effects of Sampling Bias and Model Complexity on the Predictive Performance of MaxEnt Species Distribution Models. PLoS ONE, 8, e55158.

Visser, H. \& de Nijs, T. (2006) The Map Comparison Kit. Environmental Modelling \& Software, 21, 346-358.

Warren, D.L. \& Seifert, S.N. (2011) Ecological niche modeling in Maxent: the importance of model complexity and the performance of model selection criteria. Ecological Applications, 21, 335-342.

Yackulic, C.B., Chandler, R., Zipkin, E.F., Royle, J.A., Nichols, J.D., Campbell Grant, E.H., Veran, S. (2013) Presence-only modelling using MAXENT: when can we trust the inferences? Methods Ecol. Evol. 4, 236-243. 
441 Tables

442

443 Table 1. Number of articles published during the years 2013, 2014 and 2015 available through

444 the Web of Knowledge Databases. Articles are presented per year and sample size. *Only

445 articles with sample size $\leq 90$ were used for the analyses. No info refers to articles that do not

446 provide information about the sample size used for modelling.

\begin{tabular}{ccccc}
\hline Year & $\begin{array}{c}\text { Total } \\
\text { Articles }\end{array}$ & $\begin{array}{c}\text { Articles } \\
(\mathrm{n}>90)\end{array}$ & $\begin{array}{c}\text { Articles* } \\
(\mathrm{n} \leq 90)\end{array}$ & $\begin{array}{c}\text { Articles } \\
\text { (no info) }\end{array}$ \\
\hline 2013 & 246 & 176 & 65 & 5 \\
2014 & 285 & 187 & 92 & 6 \\
2015 & 285 & 186 & 87 & 12 \\
Total & 816 & 549 & 244 & 23 \\
\hline
\end{tabular}

447

448

449

450

451

452

453

454

455

456

457

458

459

460

461

462

463 
464 Table 2. Estimation of resulting differences when using MaxEnt's default parameters or a best 465 model approach for modelling species distribution. Spatial correlation values are based in the 466 spatial correlation analysis of MaxEnt's logistic output. Fuzzy kappa was calculated after 467 applying the 10 percentile training presence logistic threshold to generate the species distribution 468 maps. Area values are based on binary maps generated after applying the 10 percentile training 469 presence logistic threshold. Best model parameters represent the combination of feature classes 470 and regularization multipliers of the model identified as of best performance for each study case.

\begin{tabular}{|c|c|c|c|c|c|c|c|c|c|}
\hline \multirow{2}{*}{$\begin{array}{c}\text { Sample } \\
\text { Size }\end{array}$} & \multirow{2}{*}{$\begin{array}{c}\text { Spatial } \\
\text { Correlation }\end{array}$} & \multirow{2}{*}{$\begin{array}{l}\text { Fuzzy } \\
\text { Карра }\end{array}$} & \multicolumn{2}{|c|}{ Area $(\mathrm{Km} 2)$} & \multicolumn{2}{|c|}{ Area $(\mathrm{Km} 2)$} & \multirow{2}{*}{$\begin{array}{c}\text { Shared / } \\
\text { not Shared } \\
\text { ratio }\end{array}$} & \multirow{2}{*}{$\begin{array}{l}\text { Best Model } \\
\text { Parameters }\end{array}$} & \multirow[b]{2}{*}{ Source } \\
\hline & & & Default & $\begin{array}{c}\text { Best } \\
\text { Model }\end{array}$ & Shared & $\begin{array}{c}\text { Not } \\
\text { Shared }\end{array}$ & & & \\
\hline 7 & 0.856 & 0.864 & 144129 & 447092 & 142612 & 0.466 & 0.466 & $\mathrm{~T} 2$ & Carvalho et al. 2015 \\
\hline 8 & 0.957 & 0.799 & 76 & 66 & 66 & 6.600 & 6.600 & Q5 & Fois et al. 2015 \\
\hline 9 & 0.905 & 0.797 & 15907 & 9771 & 9212 & 1.270 & 1.270 & LQP5 & Chunco et al. 2013 \\
\hline 10 & 0.943 & 0.781 & 861 & 1939 & 843 & 0.758 & 0.758 & Q1 & Alfaro Saiz et al. 2015 \\
\hline 11 & 0.992 & 0.943 & 122415 & 149775 & 121283 & 4.094 & 4.094 & $\mathrm{~L} 1$ & Chetan et al. 2014 \\
\hline 12 & 0.983 & 0.841 & 428209 & 551196 & 425674 & 3.324 & 3.324 & $\mathrm{~L} 2$ & Palma Perez 2013 \\
\hline 12 & 0.836 & 0.906 & 175166 & 174543 & 156798 & 4.342 & 4.342 & TQ5 & Pendersen et al. 2014 \\
\hline 13 & 0.960 & 0.843 & 33421 & 26169 & 24317 & 2.219 & 2.219 & TQ2 & Alamgir et al. 2015 \\
\hline 13 & 0.995 & 0.965 & 22013 & 26445 & 21820 & 4.528 & 4.528 & LQ1 & Mweya et al. 2013 \\
\hline 14 & 0.948 & 0.916 & 363 & 907 & 353 & 0.625 & 0.625 & LQP1 & Meyer et al. 2014 \\
\hline 15 & 0.967 & 0.900 & 5004 & 8845 & 4991 & 1.291 & 1.291 & $\mathrm{QH} 2$ & Urbani et al. 2015 \\
\hline 16 & 0.769 & 0.652 & 13466 & 28948 & 12848 & 0.768 & 0.768 & LQPT5 & De Castro et al. 2014 \\
\hline 26 & 0.865 & 0.847 & 5655316 & 7383714 & 5003914 & 1.651 & 1.651 & QP1 & Chlond et al. 2015 \\
\hline 26 & 0.945 & 0.705 & 32020 & 36420 & 28695 & 2.597 & 2.597 & $\mathrm{~L} 2$ & Simo et al. 2014 \\
\hline 31 & 0.937 & 0.879 & 243764 & 248513 & 196113 & 1.960 & 1.960 & PT1 & Orr et al. 2014 \\
\hline 49 & 0.962 & 0.880 & 135239 & 103330 & 100192 & 2.624 & 2.624 & PT1 & Hu et al. 2015 \\
\hline 54 & 0.945 & 0.858 & 2491722 & 1723084 & 1598103 & 1.569 & 1.569 & LQPT1 & Confiti et al. 2015 \\
\hline 55 & 0.841 & 0.863 & 1649518 & 1570127 & 1362351 & 2.753 & 2.753 & TQ2 & Vergara et al. 2015 \\
\hline 58 & 0.827 & 0.862 & 5822694 & 5370521 & 4439531 & 1.918 & 1.918 & $\mathrm{~T} 1$ & Aguilar et al. 2015 \\
\hline 76 & 0.934 & 0.858 & 3904018 & 3700108 & 3406765 & 4.309 & 4.309 & TQ1 & Yu et al. 2014 \\
\hline
\end{tabular}

471

472

473

474

475

476

477

478

479

480

481

482

483

484

485

Figures 
486

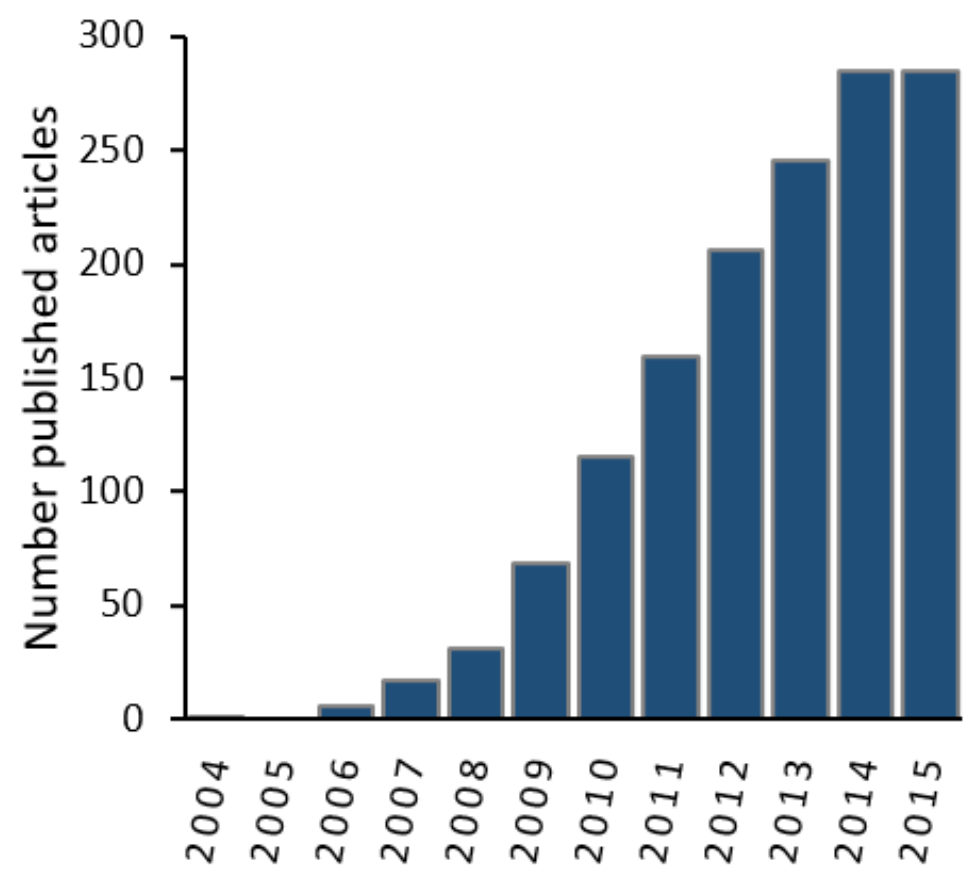

488 Figure 1. Number of published articles (2004-2015) containing both "MaxEnt" and "species distribution" within the topic in the Web of Knowledge Databases (see methods section for databases details) 


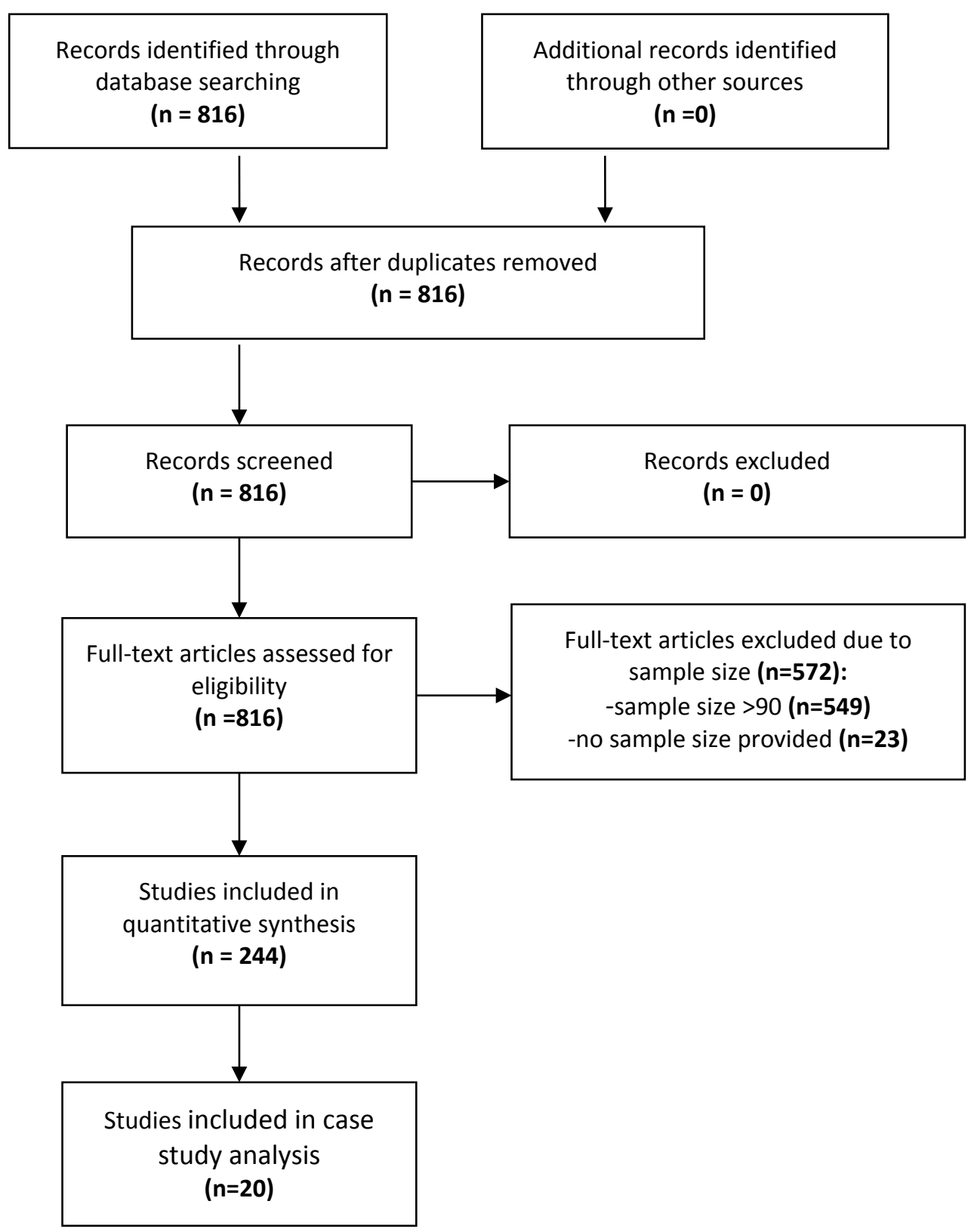

502

503

504

505

506

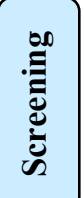

507

508

509

510

511

512

513

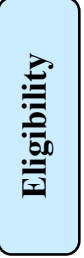

514

515

516

517

518

519

520

521

522

523

524

525

526

527

528

Figure 2. PRISMA flow diagram of the used search protocol following Moher et al. 2009. 
FEATURE CLASSES

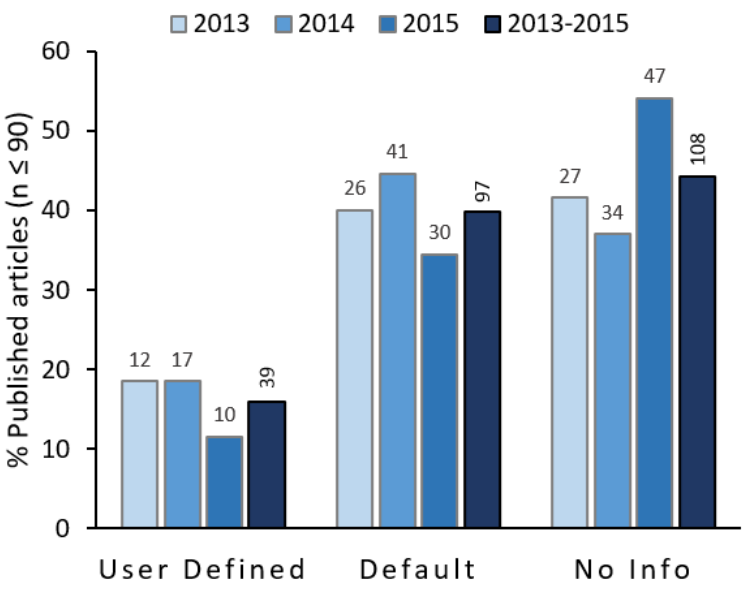

REGULARIZATION MULTIPLIER

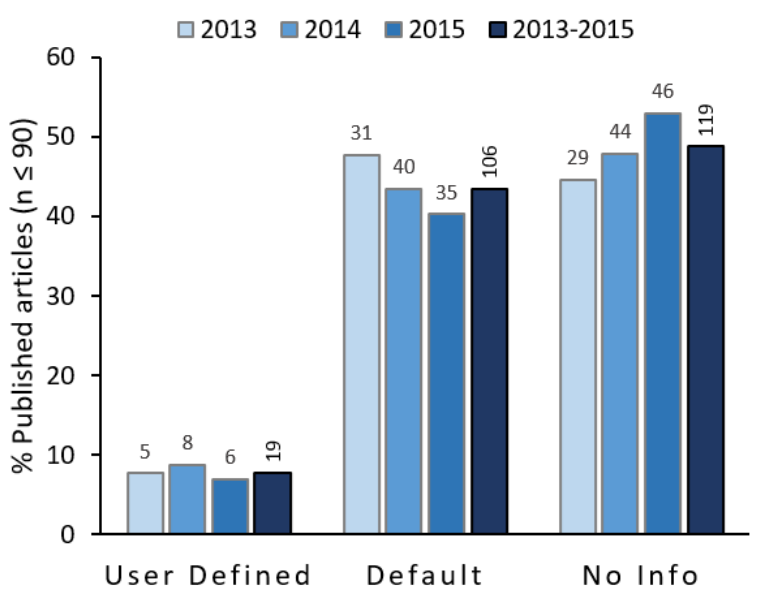

530

Figure 3. Feature classes and regularization multipliers reported to be used for modelling in the analyzed articles. Columns show the percentage of articles using user-defined, software default, and articles not providing information. Numbers on top of columns represent the number of articles pertaining to each category per year. Columns on the right of each category show the percentage and number of articles for the 2013-2015 period.

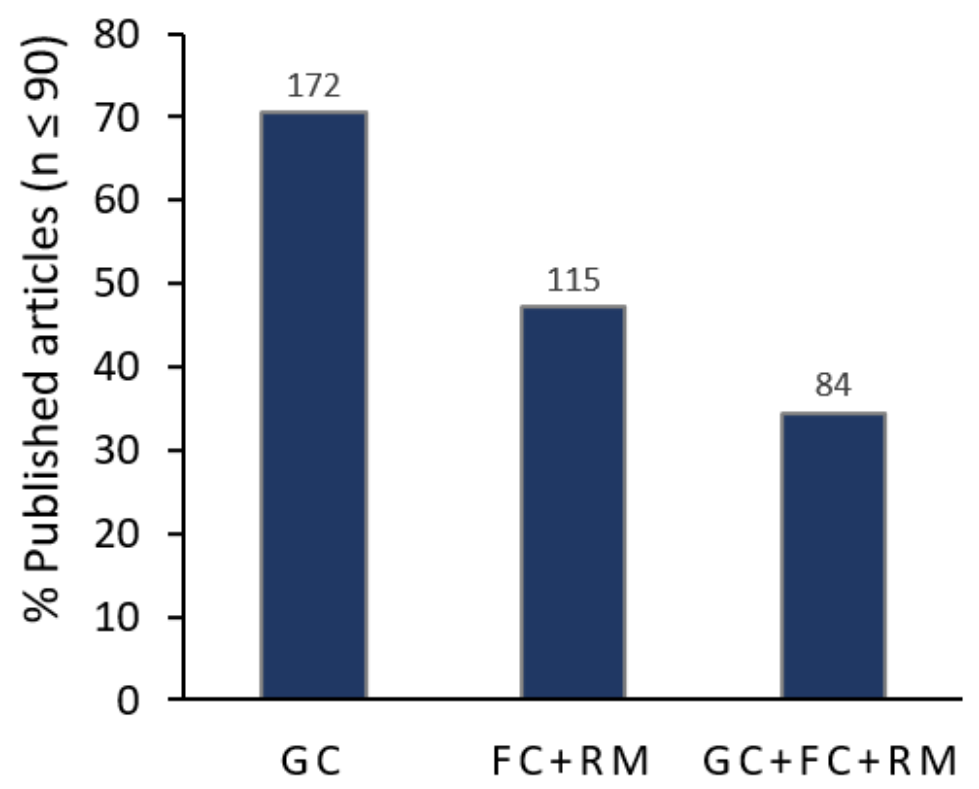

Figure 4. Replicability of the modelling process performed in analyzed articles. Columns show the percentage of articles providing information about GC: geographical coordinates, FC: feature classes, RM: regularization multiplier. Numbers above columns report the number of articles pertaining to each category. Only articles providing information regarding the three inputs (i.e. $\mathrm{GC}+\mathrm{F}+\mathrm{RM}$ column) are considered to provide enough information for replicating the modelling process. 